\title{
Fracturas torácicas y lumbares en los adolescentes. Estudio de ocho años
}

\author{
Félix Adolfo Sánchez-Chávez' y Jorge Arturo Cabrera-Escamilla² \\ 'Departamento de Cirugía de Columna, Hospital de Traumatología y Ortopedia No 21, Instituto Mexicano del Seguro Social (IMSS), Monterrey, N.L.; \\ ${ }^{2}$ Departamento de Cirugía de Columna, Hospital de Traumatología Victorio de la Fuente Narváez, IMSS, Ciudad de México. México
}

\begin{abstract}
Resumen
Método: Con el objetivo de conocer las características de estas lesiones en nuestro medio y compararlas con las de los adultos, se realiza un estudio transversal y descriptivo de los adolescentes entre 12 y 18 años de edad que se hospitalizaron por fractura torácica y lumbar en dos centros de alto nivel de traumatología en nuestro país durante 8 años. A las variables estudiadas se les aplicó análisis estadístico descriptivo y de correlación. Resultados: Se encontraron datos significativos al asociar las causas del daño con lesiones asociadas $(p=0.006)$ y al asociar el daño neurológico inicial con el daño neurológico final $(p=0.000$ ). Discusión: Se registraron siete adolescentes con 10 fracturas por arma de fuego, como causa rara de lesión. Se encontró un aumento en la frecuencia de las fracturas torácicas y lumbares en los adolescentes conforme aumenta la edad. Las fracturas torácicas y lumbares causadas por accidentes viales se asocian con lesiones en tórax y en abdomen, y cuando son causadas por caídas se asocian con fractura en extremidad inferior. Además, el 13.5\% de los casos presentaron cambios significativos hacia la mejoría en el daño neurológico inicial.
\end{abstract}

PALABRAS CLAVE: Fractura torácica. Fractura lumbar. Fracturas en adolescentes.

\begin{abstract}
Methods: In order to know the characteristics of these lesions in our environment and compare with those the adults, a transversal and descriptive study of adolescents between 12 and 18 years who were hospitalized for thoracic and lumbar fracture in two centers of high level trauma in our country for 8 years was performed. The variables studied were applied statistical analysis descriptive and correlation. Results: They were found significant date by associating the causes of damage with associated lesions $(p=0.006)$ and the initial neurological damage associated with the final neurological damage $(p=0.000)$. Discussion: They were registered seven teenagers with 10 fractures firearm, as rare cause of injury. An increase in the frequency of thoracic and lumbar fractures in adolescents with increasing age was found. The thoracic and lumbar fractures in adolescents caused by road accidents are associated with lesions in the chest and abdomen. And when are caused by falls are associated with lower extremity fractures. In addition, $13.5 \%$ of cases showed significant changes toward improvement in the initial neurological damage.
\end{abstract}

KEY WORDS: Thoracic fracture. Lumbar fracture. Fractures in adolescents.

\author{
Correspondencia: \\ Félix Adolfo Sánchez-Chávez \\ Avda. Pino Suárez y Avda. 15 de Mayo \\ Col. Centro \\ C.P. 64000 , Monterrey, N.L., México \\ E-mail: drfasanchez@hotmail.com
}

Fecha de recepción: 05-08-2016

Fecha de aceptación: 08-09-2016

DOI:10.24875/GMM.17002809

Gac Med Mex. 2017;153:672-676
Contents available at PubMed
www.gacetamedicademexico.com 


\section{Introducción}

El daño a la columna vertebral en los pacientes pediátricos es raro, con incidencia del 1 al 2\%1. Puede aparecer de manera aislada o asociado a otro tipo de daño, como a la médula espinal, traumatismo craneal, en extremidades y en otros órganos ${ }^{2}$. Es producido por traumatismos de alta energía, como sucede en los accidentes viales y en las caídas, pero cuando se presentan en niños pequeños se debe sospechar abuso infantili ${ }^{3,4}$. El daño a la columna por proyectil de arma de fuego (PPAF) suele verse en la población militar, presenta un incremento actual en la población civil, debido a la fácil disponibilidad para la obtención de armas de manera no autorizada o ilegal ${ }^{5,6}$. Cerca del $32 \%$ de los pacientes con lesiones neurológicas asociadas a la fractura de columna no son diagnosticados al momento del daño, principalmente en pacientes que presentan disminución del nivel de consciencia por traumatismo craneal y en pacientes con daños asociados en la pelvis y en las extremidades ${ }^{7,8}$.

La tomografía computarizada ha reemplazado a las radiografías convencionales como la imagen de elección primaria en los pacientes traumatizados, debido a su alta agudeza diagnóstica y a su costo-beneficio, reduciendo el número excesivo de radiografías tomadas $^{9-11}$. La columna vertebral en los niños tiene más tejido blando y más periostio comparados con los adultos, resultando en un mayor potencial para la reparación de sus lesiones, la remodelación ósea, la cicatrización de los ligamentos dañados y la recuperación del daño neurológico ${ }^{12-14}$. Una fractura de columna estable es una fractura con menos de $20^{\circ}$ de deformidad en cifosis, con menos del $50 \%$ de pérdida de altura del cuerpo vertebral, con preservación de su columna posterior y sin déficit neurológico ${ }^{15}$. La inestabilidad se presenta cuando existe una fractura con cifosis progresiva de más de $20^{\circ}$, pérdida de altura del cuerpo vertebral dañado de más del $50 \%$ y fragmentos en el canal vertebral, asociado a déficit neurológico ${ }^{16-18}$. La estabilización quirúrgica de la fractura dentro de las primeras 24 horas reduce las complicaciones relacionadas con el reposo ${ }^{19}$, y la utilización de tornillos pediculares ha mostrado ser más efectiva como método de fijación espinal tanto en los niños como en los adultos $^{20}$. La región torácica (T2-T10) representa el sitio de mayor afección en los niños ${ }^{21}$. La unión toracolumbar (T12, L1 y L2) es la mayormente afectada en los adolescentes y los adultos ${ }^{22,23}$. Las clasificaciones propuestas por Magerl y Frankel resultan adecuadas para la evaluación del daño morfopatológico de la fractura de columna y del daño neurológico asociado ${ }^{24,25}$.

Se realizó el presente estudio para conocer las características de este tipo de lesiones en nuestro medio. Nos planteamos como objetivo identificar las características distintivas de las fracturas torácicas y lumbares en los adolescentes e identificar diferencias con los adultos.

\section{Métodos}

Pacientes adolescentes de entre 12 y 18 años hospitalizados en el servicio de columna en dos centros de alto nivel de atención, la Unidad Médica de Alta Especialidad Hospital de Traumatología y Ortopedia No 21 del Instituto Mexicano del Seguro Social (IMSS) en la ciudad de Monterrey, Nuevo León, y el Hospital de Traumatología Victorio de la Fuente Narváez, del IMSS, en Ciudad de México, fueron seleccionados como muestra los que ingresaron con el diagnóstico de fractura torácica y lumbar, del año 2006 al 2013. Se realizaron análisis estadísticos descriptivos y de correlación a las variables estudiadas con el auxilio del programa estadístico SPSS versión 22. Además de realizar estadística descriptiva, la estadística inferencial o de asociación se realizó utilizando la prueba no paramétrica ji al cuadradopara comparar proporciones, considerando una asociación estadísticamente significativa cuando el valor de p fue menor de 0.05.

\section{Resultados}

Se incluyó a 96 adolescentes, encontrando predominio del sexo masculino en el $66.7 \%$ de los casos, una edad media de $16.4 \pm 1.6$ años y una moda de 18 años (Fig. 1). La principal causa de lesión fueron las caídas de altura, en un $57.3 \%$, seguidas de los accidentes viales en un $34.4 \%$. También se encontraron causas raras, como son fracturas de columna en adolescentes por PPAF en el 7.3\% de los casos (Fig. 2).

Las lesiones asociadas a la fractura vertebral se presentaron en 40 pacientes (41.7\%), predominando el traumatismo craneal en el $12.5 \%$ y las fracturas en la extremidad inferior en un $10.4 \%$, mientras que 56 pacientes $(58.3 \%)$ no tuvieron lesiones asociadas (Tabla 1). Se encontraron datos significativos por medio del análisis estadístico de contraste $\chi^{2}\left(\chi^{2}=32.001\right.$; $\mathrm{gl}=15 ; p=0.006)$ al relacionar la causa de la lesión y las lesiones asociadas. Las fracturas de columna producidas por los accidentes viales se asocian con lesiones en el tórax y con lesiones abdominales de 


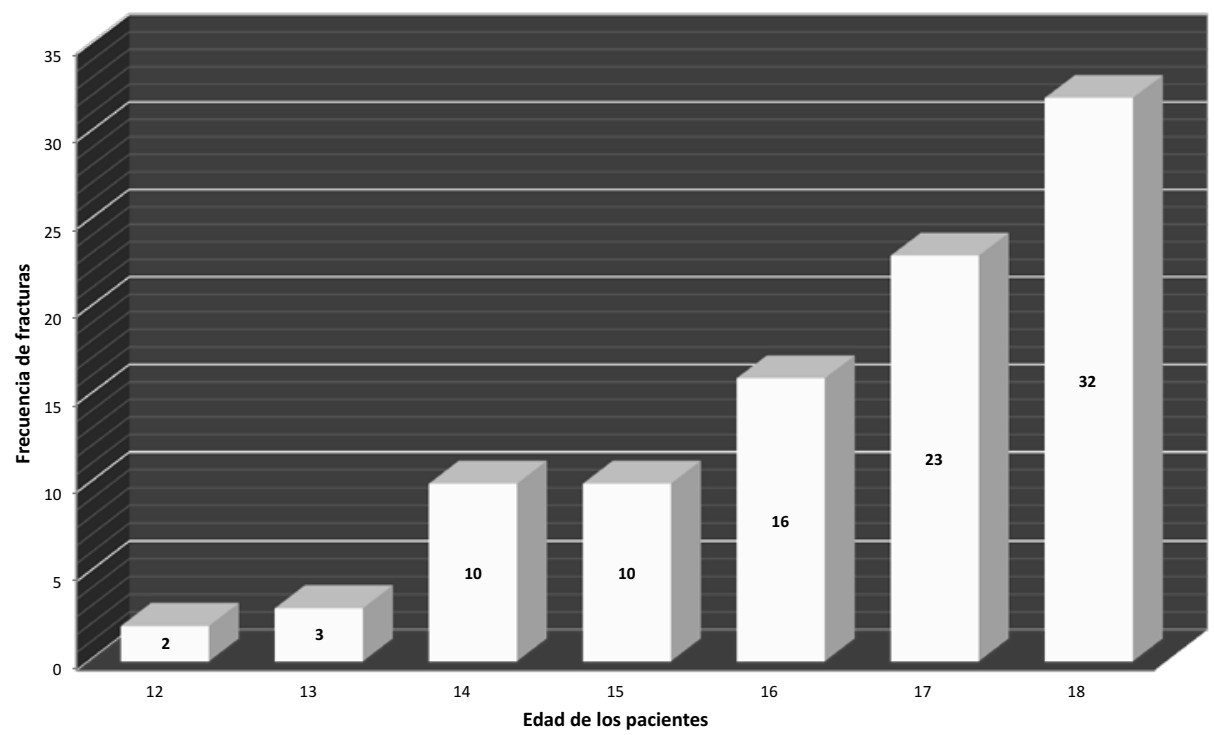

Figura 1. Distribución de las fracturas por edad.

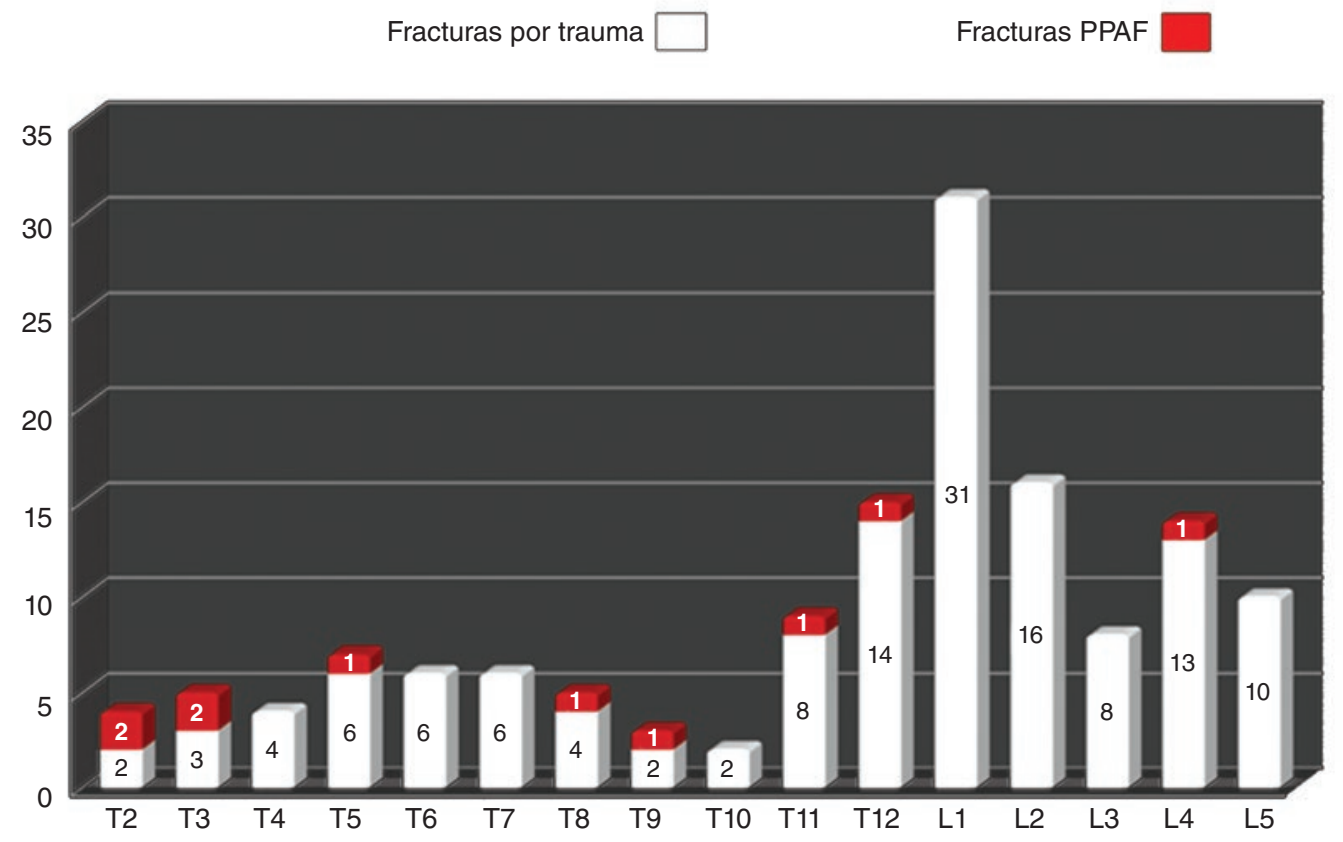

Figura 2. Distribución de las fracturas por nivel de afectación.

manera significativa. Las fracturas de columna producidas por las caídas se asocian significativamente con daño a las extremidades inferiores (Tabla 2).

Se registraron 64 casos con fracturas en un solo nivel, 29 casos con fracturas en dos niveles y 5 casos con fracturas en tres niveles. El nivel vertebral más frecuentemente afectado fue $L 1$, en el $29.2 \%$, seguido por $\mathrm{L} 2$ en el $12.5 \%$, T12 en el $10.4 \%$ y $L 4$ en el $9.4 \%$ (Fig. 2). Se encontraron lesiones tipo A de Magerl en el $40.6 \%$ de los pacientes, tipo B en el $26 \%$ y tipo C también en el $26 \%$. Sumando un total de 135 fracturas vertebrales en 96 pacientes, 125 fueron fracturas traumáticas y 10 fueron por PPAF. Se trataron con cirugía 86 casos y 10 de manera conservadora. El $90.7 \%$ de los casos que se operaron fueron resueltos con un solo abordaje posterior, el $8.1 \%$ requirieron abordaje combinado posterior-anterior y el $1.2 \%$ requirió de un solo abordaje anterior.

El déficit neurológico asociado a la fractura vertebral se presentó en 32 pacientes (33.3\%) de manera inicial, 
Tabla 1. Lesiones asociadas

\begin{tabular}{|c|c|c|}
\hline TRAUMA CRANEO-ENCEFÁLICO & & 12 casos \\
\hline Trauma Craneo-Encefálico & 12 casos & \\
\hline \multicolumn{2}{|l|}{ LESIÓN EN TÓRAX } & 9 casos \\
\hline Fracturas costales & 1 caso & \\
\hline Hemo-neumotórax & 6 casos & \\
\hline Neumotórax & 2 casos & \\
\hline \multicolumn{2}{|l|}{ LESIÓN EN ABDOMEN } & 5 casos \\
\hline Lesión de Bazo & 2 casos & \\
\hline Lesión Hígado & 2 casos & \\
\hline Fractura de Pelvis & 1 caso & \\
\hline \multicolumn{2}{|l|}{ EXTREMIDAD SUPERIOR } & 4 casos \\
\hline Fractura de Húmero & 1 caso & \\
\hline Fractura Radiocubital & 1 caso & \\
\hline Fractura de clavícula + lesión plexo & 1 caso & \\
\hline Fractura de Radio & 1 caso & \\
\hline \multicolumn{2}{|l|}{ EXTREMIDAD INFERIOR } & 10 casos \\
\hline Fractura de Fémur & 1 caso & \\
\hline Fractura Tibioperonea & 2 casos & \\
\hline Fractura de Tobillo & 4 casos & \\
\hline Fractura de Calcáneo & 2 casos & \\
\hline Fractura de Astrágalo & 1 caso & \\
\hline \multicolumn{2}{|l|}{ SIN LESIÓN ASOCIADA } & 56 casos \\
\hline
\end{tabular}

Tabla 2. Tabla de asociación estadística entre las variables

\begin{tabular}{|c|c|c|}
\hline Variables & Prueba $\chi^{2}$ & Interpretacion \\
\hline Sexo y edad & $p=0.366$ & \\
\hline Mecanismo de lesión y lesiones asociadas & $p=0.006$ & $\begin{array}{l}\text { Los accidentes viales se asocian con lesiones en el tórax y en el } \\
\text { abdomen } \\
\text { Las caídas se asocian con lesiones en la extremidad inferior }\end{array}$ \\
\hline Mecanismo de lesión y sexo & $p=0.501$ & \\
\hline Mecanismo de lesión y edad & $p=0.551$ & \\
\hline Daño neurológico inicial y daño neurológico final & $p=0.000$ & $\begin{array}{l}\text { En el } 86 \% \text { de los casos la evaluación neurológica no cambia, es } \\
\text { la misma al inicio que al final del tratamiento } \\
\text { En el } 13.5 \% \text { de los casos existen cambios en la evaluación } \\
\text { neurológica hacia la mejoría }\end{array}$ \\
\hline Clasificación de la lesión y edad & $p=0.076$ & \\
\hline Lesión única, doble o triple y edad & $p=0.999$ & \\
\hline
\end{tabular}

y 64 casos (66.6\%) no presentaron déficit. Doce pacientes se presentaron con lesión medular completa 0 Frankel A, y 20 pacientes con lesiones intermedias: Frankel B 1 paciente, Frankel C 11 pacientes y Frankel D 8 pacientes. Una vez consolidada la lesión o la artrodesis, aproximadamente 4 meses después de tratada la fractura, el déficit neurológico persistió en 21 pacientes (21.9\%), 11 con lesión medular completa o Frankel A y 10 con grados intermedios de lesión neurológica: Frankel C 9 pacientes y Frankel D 1 paciente. Al realizar pruebas de asociación estadística entre la variable daño neurológico inicial y la variable daño neurológico final, 
resultó ser altamente significativa $\left(\chi^{2}=159.037 ; \mathrm{gl}=12\right.$; $p=0.000$ ) (Tabla 1). En 83 casos (86.5\%) se identificó los mismos resultados en la evaluación neurológica inicial y final. Los 13 casos restantes (13.5\%) tuvieron cambios neurológicos significativos hacia la mejoría. Los siete pacientes lesionados por PPAF permanecieron sin cambios neurológicos entre las evaluaciones inicial y final: Frankel A 3 pacientes, Frankel $C 1$ paciente, Frankel D 1 paciente y Frankel E 2 pacientes.

\section{Discusión}

Describimos una frecuente aparición de lesiones asociadas a la fractura vertebral en los pacientes pediátricos, ya que el $41.7 \%$ de los estudiados presentaron lesiones asociadas, mientras que el $58.3 \%$ restante no las presentaron. Se observa un incremento en la frecuencia de las fracturas de columna en los adolescentes conforme aumenta la edad, como también mencionan Hofbauer, et al. ${ }^{1}$ y Moller, et al. ${ }^{26}$.

Cuando la fractura de columna en los adolescentes es causada por accidente vial, existe una alta posibilidad de presentarse lesiones asociadas en el abdomen y en el tórax. $Y$ cuando la fractura es causada por caídas, existe una alta posibilidad de presentarse lesiones asociadas en las extremidades inferiores. Llama la atención las 10 vértebras fracturadas por PPAF en siete adolescentes de nuestra serie, debido al aumento de la violencia que se vive en las grandes ciudades ${ }^{5,6}$.

El $13.5 \%$ de los pacientes del estudio que sufrieron daño neurológico inicial presentaron cambios significativos hacia la mejoría, a diferencia de lo que sucede en los pacientes adultos. Los niños tienen un gran potencial para recuperarse del daño neurológico ${ }^{14}$.

En el presente estudio no se encontró un tipo predominante de lesión, al contrario de los adultos, en los que predominan las lesiones tipo $\mathrm{A}^{24}$. Solo un caso de nuestra serie ameritó ser resuelto con un solo abordaje anterior; el $90.7 \%$ de los casos fueron resueltos con un solo abordaje posterior. Debido a que el disco y los ligamentos absorben las fuerzas aplicadas al hueso, las fracturas con estallamiento completo son raramente vistas en los niños ${ }^{19}$. Los adolescentes con fractura torácica y lumbar sí pueden ser tratados como los adultos, pero a diferencia de ellos solo con el abordaje posterior se pueden tratar la gran mayoría de estas fracturas.

\section{Conflictos de intereses}

Los autores declaran no tener ningún conflicto de intereses.
La investigación no contó con ayuda financiera ni de cualquier tipo, y no tiene vínculos financieros con ninguna compañía.

\section{Bibliografía}

1. Hofbauer M, Jaindl M, Lee Hochtl L, et al. Spine injuries in polytraumatized pediatric patients: characteristics and experience from a level 1 trauma center over two decades. J Trauma Acute Care Surg. 2012;73:156-61.

2. Moradi-Lakeh M, Rasouli M, Vaccaro A, et al. BMC Public Health. 2011;11:789.

3. Jansson KA, Blomqvist $P$, Svedmark $P$, et al. Thoracolumbar vertebral fractures in Sweden: an analysis of 13,496 patients admitted to hospital. Eur J Epidemiol. 2010;25:431-7.

4. Habert J, Haller JO. latrogenic vertebral body compression fracture in a premature infant caused by extreme flexion during positioning for a lumbar puncture. Pediatr Radiol. 2000;30:410-1.

5. Jaiswal M, Mittal RS. Concept of gunshot wound spine. Asian Spine J. 2013;7:359-64.

6. Bordon G, Girona B. Gunshot wound in lumbar spine with intradural location of a bullet. Case Reports in Orthopedics. 2014; 1-4. Disponible en: http://dx.doi.org/10.1155/2014/698585

7. De Amoreira RG, Nadal LG. Spinal cord trauma in children under 10 years of age: clinical characteristics and prevention. Childs Nerv Syst. 2012;28:1919-24.

8. Junkins EP, Stotts A, Santiago R, et al. The clinical presentation of pediatric thoracolumbar fractures: a prospective study. J Trauma. 2008;65:1066-71.

9. Bensch FV, Kiuru MJ, Koivikko MP, et al. Spine fractures in falling accidents: analysis of multidetector CT findings. Eur Radiol. 2004;14:618-24.

10. Bensch FV, Koivikko MP, Koskinen SK. MDCT findings in sports and recreational accidents. Acta Radiologica. 2011;52:1107-12.

11. Jiménez RR, DeGuzmán NA, Shiran S, et al. CT versus plain radiographs for evaluation of $c$-spine in young children: do benefits outweigh risks? Pediatr Radiol. 2008;38:635-44.

12. Mortazavi MM, Dogan S, Civelek E, et al. Pediatric multilevel spine injuries: an institutional experience. Childs Nerv Syst. 2011;27:1095-100.

13. Sieradzki JP, Sarwark JF. Thoracolumbar fracture-dislocation in child abuse: case report, closed reduction technique and review of the literature. Pediatr Neurosurg. 2008;44:253-7.

14. Basu S. Spinal injuries in children. Frontiers in Neurology. 2012;3:1-15. Disponible en: http://www.frontiersin.org/SpinalcordMedicine/archive

15. Thomas KC, Lalonde F, ONeil J, et al. Multiple level thoracolumbar burst fractures in teenaged patients. J Pediatr Orthop. 2003;23:119-23.

16. Van der Roer N, de Lange ESM, Bakker FC, et al. Management of traumatic thoracolumbar fractures: a systematic review of the literature. Eur Spine J. 2005;14:527-34.

17. Van Goethem JW, Maes M, Ozsarlak O, et al. Imaging in spinal trauma. Eur Radiol. 2005;15:582-90.

18. Wang ST, Ma HL, Liu ChL, et al. Is fusion necessary for surgically treated burst fractures of the thoracolumbar and lumbar spine? Spine. 2006;31:2646-52.

19. Pakzad H, Roffey DM, Knight $\mathrm{H}$, et al. Delay in operative stabilization of spine fractures in multitrauma patients without neurologic injuries: effects on outcomes. Can J Surg. 2011;54:270-6.

20. Mueller TL, Miller NH, Baulesh DM, et al. The safety of spinal pedicle screw in children ages 1 to 12. Spine J. 2013;13:894-901.

21. Reddy SP, Junewick JJ, Backstrom JW. Distribution of spinal fractures in children: does age, mechanism of injury, or gender play a significant role? Pediatr Radiol. 2003;33:776-81.

22. Knop Ch, Kranabetter T, Reinhold M, et al. Combined posterior-anterior stabilization of thoracolumbar injuries utilising a vertebral body replacing implant. Eur Spine J. 2009;18:949-63.

23. Steinmetz MP, Resnick DK. Thoracolumbar fractures. Contemporary Neurosurgery 2006;28:1-6.

24. Aebi M. Classification of thoracolumbar fractures and dislocations. Eur Spine J. 2010;19(Suppl 1):S2-7.

25. Frankel HL, Hancock DO, Hyslop G, et al. The value of postural reduction in the initial management of closed injuries of the spine with paraplegia and tetraplegia. I. Paraplegia. 1969;7:179-92.

26. Moller A, Hasserius R, Besjakov J, et al. Vertebral fractures in late adolescence: a 27 to 41 year follow-up. Eur Spine J. 2006;15: 1247-54 